\title{
Electrical Transport and Current Conduction Mechanisms in ZnO/Si Heterojunction Diode
}

\author{
M. Benhaliliba ${ }^{1,2, *}$ \\ ${ }^{1}$ Film Device Fabrication-Characterization and Application FDFCA Research Group USTOMB, 31130, Oran, \\ Algeria \\ ${ }^{2}$ Physics Faculty, USTOMB University POBOX 1505 31130, Mnaouer Oran Algeria
}

\begin{abstract}
The objective of this research is the relevant equations of electrical transport inside a junction device based on metallic oxides like zinc oxides retained on silicon substrate by spray pyrolysis process. Many characteristics, such as the heterojunction diode's non-ideal conduct, electronic conduction of electrons and gaps in the conduction and valence bands, charge carriers caught by trap centers, hopping conduction, and tunnel effect, are used in various conduction processes at electronic junctions. Poole-Frenkel (PF) emission, Schottky emissions, and trap aided tunneling are some of the other conduction mechanisms examined inside forward/reverse bias for $\mathrm{ZnO} / \mathrm{Si}$ heterojunctions (TAT). This article also confirms, addresses, and elucidates the effect of temperature on the $\mathrm{I}-\mathrm{V}$ properties of $\mathrm{ZnO} / \mathrm{Si}$.

https://ecee.colorado.edu/ bart/book/book/chapter3/ch3 9.htm
\end{abstract}

Keywords: Conduction mechanisms, ZnO/Si, Thermoionic emission, Poole-Frenkel (PF) emission.

\section{INTRODUCTION}

To replace III-V semiconductors with wide band gap II-VI semiconductors $(\mathrm{Eg}>3 \mathrm{eV})$ such as tin and zinc oxides, researchers have conducted multiple studies to identify oxides with a large band gap, sufficient transparency in the Vis-IR bands, and appropriate electrical conductivity. Following that, these semiconductor materials may be used in electrical and optoelectronic device applications [1, 2]. Understanding transport current and conduction is one of the most important phenomena explored by researchers in the field of microelectronic devices. If a device is heated, are I-V characteristics and related parameters affected by temperature? Are the conduction mechanism and electrical transport in such device influenced by temperature?

This device-based zinc oxide research is being carried out for this aim. Ohmic conduction, Schottky emission, thermionic emission, tunneling conduction, hopping conduction, Frenkel-Poole emission, trapassisted electron transport, and space charge limited current are among the conduction mechanisms inside the device [3-9]. The Poole-Frenkel phenomenon, also known as Frenkel-Poole emission in condensed matter, is a model for trap-assisted electron transport in an electrical insulator $[9,10]$. The following technique

*Address correspondence to this author at the Film Device FabricationCharacterization and Application FDFCA Research Group USTOMB, 31130, Oran, Algeria;

E-mail: mbenhaliliba@gmail.com allows charge carriers such as electrons to trave slowly through an insulator. In general, electrons are locked in localized states. Changes in heat activity inside a semiconductor provide an electron with enough energy to leave its confined state and transfer to the conduction band on an irregular basis (CB). The free charge carrier is allowed to move within the crystal for a short period of time in this condition before relaxing to an unoccupied localized state.

The Poole-Frenkel effect demonstrates how an electron does not require enough heat energy to be pushed into the $\mathrm{CB}$ when a big enough electric field is present. The Poole-Frenkel effect is theoretically very similar to the Richardson-Schottky effect. This is the result of the electric interaction with the field at the metal-insulator boundary lowering the metal-insulator energy barrier. The conductivity resulting from the PF effect is discovered in the presence of bulk-limited conduction, but it is contact limited from RichardsonSchottky [4]. Oxide-based heterojunctions are manufactured and electrical transport magnitudes are studied in this study. The oxide layer has been covered with a metallic contact to investigate the conduction mechanism behavior of such heterostructures under temperature conditions.

Furthermore, the current and capacitance versus bias voltage are measured in special conditions of temperature. A study of oxides and detailed conduction mechanisms are presented is a novelty of this research. 


\section{THEORY OF CONDUCTION MECHANISM IN ELECTRONIC DEVICES}

In this part, the hypothesis and models of electrical transport are depicted. It is reported for that two principal classes of electrical transport can be occurred; bulk and electrode limited. Bulk limited is that mechanism where the bulk of the insulating material has a dominating role Poole-Frenkel and hopping. While electrode limited are those where electrode material has a dominating role tunneling (direct, Fowler Nordheim (F-N), trap assisted tunneling TAT) [11, 12].

The tunneling current is an exponential function of tunneling distance (a) expresses as;

$$
J=A \exp (-B a)
$$

Where $A$ is pre-exponential parameter and $B$ is a coefficient $\left(\mathrm{m}^{-1}\right)$.

In F-N regime, current density obeys to following law;

$J=C E_{i}{ }^{2} \exp \left[-\frac{4\left(q \phi_{b}\right)^{3 / 2} \sqrt{m^{*}}}{3 q \hbar E_{i}}\right]$

Where $C$ in terms of charge, effective mass, height barrier, is given by;

$C=\frac{q^{3} m_{e f f}}{8 \pi m d_{\text {diel }} h q \phi_{b}}$

The basic conduction processes in insulators are as follows [13];

\subsection{Thermionic Emission}

The thermionic discharge hypothesis accepts that electrons, with an energy greater than the head of the obstruction, will cross the hindrance gave they move towards the boundary. The real state of the obstruction is thusly disregarded. The current expresses as [13];

$$
J=A^{*} T^{2} \exp \left[-\frac{q\left(\phi_{b}-\sqrt{q E_{i} / 4 \pi \varepsilon_{i}}\right.}{k T}\right]
$$

A current against voltage and temperature dependence are given by;

$$
J \alpha T^{2} \exp \left[\frac{q\left(a \sqrt{V}-\varphi_{b}\right)}{k T}\right]
$$

\subsection{Tunneling Process}

For tunneling process, current density $\mathrm{J}$ is expressed in terms of electric field, barrier height, effective electron mass as $[12,14]$;

$J \alpha E_{i}{ }^{2} \exp \left[-\frac{4\left(q \phi_{b}\right)^{3 / 2} \sqrt{2 m^{*}}}{3 q \hbar E_{i}}\right]$

Current density versus voltage in term of temperature expresses as [14];

$$
J \alpha V^{2} \exp \left(\frac{-b}{V}\right)
$$

\subsection{The Frenkel-Poole Emission}

The Frenkel-Poole emission is caused by trapped electrons emission into the conduction band. A thermal excitation can move a number of electrons from traps. For trap states with Coulomb potential type, the expression is comparable to that of the Schottky emission. It is well known that barrier height is the depth of the trap potential well. The barrier reduction is larger than in the case of Schottky emission by a factor of 2 , since the barrier dropping is twice as large due to the quasi total immobility of the positive charge. Current density $\mathrm{J}$ in terms of electric field $E_{i}$, barrier height $\varphi_{\mathrm{b}}$ and temperature expresses as [15];

$J \alpha E_{i} \exp \left[-\frac{q\left(\phi_{b}-\sqrt{q E_{i} / \pi \varepsilon_{i}}\right)}{k T}\right]$

$J \alpha V \exp \left[\frac{q\left(-\phi_{b}+2 a \sqrt{V}\right)}{k T}\right]$

Where $a$ is equal to $\left(q / 4 \pi \varepsilon_{i} d\right)^{1 / 2}, V=E_{i} d$ is the applied voltage

In terms of temperature it is written as [16-18]

$$
J=A T^{2} \exp \left(\frac{1}{k T}\right)\left[\left(\frac{a}{E_{i} d}\right)^{0.5}-\varphi_{t}\right]
$$

Where $A$ is a constant having different values for PFE and SE, $\mathrm{J}$ is the current density, $\mathrm{T}$ is the absolute temperature, $k$ is Boltzmann constant, $E_{i}$ is the applied field in semiconductor barrier at the metalsemiconductor interface, $a$ is a constant equal to 57.7 $\mathrm{eV}, \Phi_{\mathrm{t}}$ is the trap potential 


$$
\frac{\ln J}{A T^{2}}=\left(\frac{1}{k T}\right)\left[\frac{b}{V^{0.5}}-\varphi_{t}\right]
$$

This equation is linear curve $\operatorname{Ln}\left(\mathrm{J} / \mathrm{AT}^{2}\right)$ versus $1 / \mathrm{V}^{0.5}$, $\mathrm{b}$ is constant equal to 7.59 presenting a slope of $\mathrm{b} / \mathrm{kT}$ and intercept of $-\varphi \mathrm{t} / \mathrm{k} \mathrm{T}$.

\subsection{Space Charge Limited Current SCLC}

Current density versus voltage finds an easy equation as follows [19];

$J=\frac{9 \varepsilon_{i} \mu V^{2}}{8 d^{3}}$

Where $d$ is the thickness of insulator layer, $\mu$ is the mobility, $\varepsilon_{\mathrm{i}}$ is dielectric constant of insulator,

\subsection{Ohmic Regime}

Current density in terms of temperature is given by [20];

$J \alpha E_{i} \exp \left[-\frac{\Delta E_{e}}{k T}\right]$

Where $\Delta \mathrm{E}_{\mathrm{e}}$ is an activation energy of electrons

$J \alpha V \exp \left[-\frac{c}{T}\right]$

Where $\mathrm{c}$ is a constant.

\subsection{Ionic Conduction}

The current density in terms of electric field $E_{i}$ and temperature $\mathrm{T}$ is giving by [13];

$$
\begin{aligned}
& J \alpha \frac{E_{i}}{T} \exp \left[-\frac{\Delta E_{i}}{k T}\right] \\
& J \alpha \frac{V}{T} \exp \left[-\frac{d}{T}\right]
\end{aligned}
$$

Where $\Delta E_{i}$ is activation energy of ions, $A^{*}$ effective Richardson constant,

\subsection{Fowler-Nordheim Tunneling}

Current mechanisms through materials, which do not contain free carriers, can be distinctly different from those in doped semiconductors or metals. Here, Fowler-Nordheim Tunneling, Poole-Frenkel emission as well as Space charge effects transport are discussed. While these current mechanisms are not identified with Metal-Semiconductor junctions, they are presented here since they represent unipolar current mechanisms.

Fowler-Nordheim tunneling has been studied extensively in Metal-Oxide-Semiconductor heterostructures where it has been shown to be the dominant current mechanism, particularly for thick oxides. It is explained by that quantum mechanical tunneling from the contiguous conductor into the insulator limits the current through the heterostructure. Once the carriers have tunneled into the insulator they are free to move within the valence or conduction band of the insulator. The current is deduced from WBK approximation as [15];

$J=C E_{i}{ }^{2} \exp \left[-\frac{4\left(q \phi_{b}\right)^{3 / 2} \sqrt{m^{*}}}{3 q \hbar E_{i}}\right]$

$C=\frac{q^{3} m_{e f f}}{8 \pi m d_{d i e l} h q \phi_{b}}$

$J_{F N}=C_{F N} \varepsilon_{o x}^{2} \exp \left[-\frac{4}{3} \frac{\sqrt{2 m_{o x}^{*}}}{q \hbar} \frac{\left(q \varphi_{b}\right)^{3 / 2}}{\varepsilon_{o x}}\right]$

The expression for Fowler-Nordheim tunneling implies that carriers are free to move through the insulator. Whereas this is indeed the case in thermally grown silicon dioxide it is frequently not so in deposited insulators, which contain a high density of structural defects. Silicon nitride $\left(\mathrm{Si}_{3} \mathrm{~N}_{4}\right)$ is an example of such material. The structural defects cause additional energy states close to the band edge, called traps. These traps restrict the current flow because of a capture and emission process, thereby becoming the dominant current mechanism. The current is a simple drift current described by

While the carrier density depends exponentially on the depth of the trap, which is corrected for the electric field;

$$
n=n_{0} \exp \left[\frac{-q}{k T}\left(\varphi_{B}-\sqrt{\frac{q E_{N}}{\pi \varepsilon_{N}}}\right)\right]
$$

The total current then equal

$$
J_{P F}=q n_{0} \mu \mathrm{E}_{N} \exp \left[-\frac{q}{k T}\left(\varphi_{b}-\sqrt{\frac{q \mathrm{E}_{N}}{\pi \varepsilon_{N}}}\right)\right]
$$




\subsection{Schottky Diode Current}

Generally the majority charge carriers electrons or holes produce the current over the MS semiconductor. Thus, three different mechanisms happen,

Three unmistakably various components exist: dissemination of transporters from the semiconductor into the metal, thermionic emanation of transporters over the Schottky hindrance and quantum-mechanical burrowing through the boundary. The dissemination hypothesis expects that the main impetus is circulated over the length of the consumption layer. The thermionic discharge hypothesis then again proposes that lone lively transporters, those, which have an energy equivalent to or bigger than the conduction band energy at the metal-semiconductor interface, add to the current stream. Quantum-mechanical burrowing through the hindrance considers the wave-idea of the electrons, permitting them to enter through slim boundaries. In a given intersection, a blend of each of the three components could exist. Notwithstanding, commonly one finds that just a single current system rules.

\subsection{Space Charge Limited Current SCLC}

Throught this section, that emission mechanism like Fowler Nordheim tunneling and Poole-frenkel produce very low current densities with consistently low carrier densities are demonstrated. For structures where carriers can readily enter the insulator and freely flow through the insulator.

For structures where transporters can promptly enter the protector and uninhibitedly move through the separator one tracks down that the subsequent current and transporter densities are a lot higher. The high thickness of these charged transporters causes a field slope, which restricts the current thickness. The present circumstance happens in humble doped semiconductors and vacuum tubes.

\subsection{The Barrier Height and Fermi Energy}

The barrier height, $\Phi_{\mathrm{B}}$, is defined as the potential difference between the Fermi energy of the metal and the band edge where the majority carriers reside. One finds that for an $n$-type semiconductor the barrier height is obtained from $[15,21]$;

$\varphi_{B}=\Phi_{M}-\chi$

Where $\Phi_{M}$ is the work function of the metal and $X$ is the electron affinity. The barrier height is given by the difference between the valence band edge and the Fermi energy in the metal [21];

$\varphi_{B}=\frac{E_{g}}{q}-\Phi_{M}+\chi$

\section{For p-type semiconductor}

A metal-semiconductor junction will therefore form a barrier for electrons and holes if the Fermi energy of the metal as drawn on the flat-band diagram is somewhere between the conduction and valence band edge.

In addition, the built-in potential is defined, $\mathrm{V}_{\mathrm{bl}}$, as the difference between the Fermi energy of the metal and that of the semiconductor.

Barrier height behavior and Fermi level energy

The barrier height $\Phi_{B}$ is defined as the potential difference between the fermi energy of the metal and the band edge where the common carriers reside. For n-type semiconductor $\Phi_{B}$ expresses as [21];

$\varphi_{B}=W-\chi$

Where $\mathrm{W}$ is the work function of the metal and $\chi$ is the electron affinity. For p-type case it is the difference between the valence band edge and the Fermi energy metal written as follows [21];

$\varphi_{B}=\frac{E_{g}}{q}-W+\chi$

Inside an electrical device, many conduction mechanisms might occur like;

${ }^{*}$ The recombinaison-diffusion: logl- $\mathrm{V}-\mathrm{T}$ is a linear curve for $1<n<2$, $n-T$ still a constant parameter.

*Thermionic emission: logl-V-T is a linear curve, $n-T \log I_{0}$ depends on $1 / T$ follows linear curve, $q \Phi_{b i}$ is constant and $E_{a}$ is close to $E_{g}$.

*Tunnel effect by step: logl-V-T presents a linear curve, $1<n<2$, nT is constant for any temperature while $q \Phi_{b i}$ is parameter which depends on $T$.

*Field assisted thermionic emission: logl- $\mathrm{V}-\mathrm{T}$ is a linear curve, $1<n<2, \log I_{0}$ depends linearly on $n / T$, ideality factor is a function of $T$ and $E_{a}<E_{g}$.

*Recombination $\log \mathrm{I}_{0}$ is a function of $1 / \mathrm{T}, \log \mathrm{I}_{0}-\mathrm{T}$ is linear $n=2$ and $E_{a}=E_{g} / 2$. 
Injection of minority charge carriers: $\log \mathrm{l}-\mathrm{V}-\mathrm{T}$ is a linear curve, $n=1, \log I_{0}$ is a linear function of $1 / T$.

\section{${ }^{*}$ Transport mechanism in $\mathrm{ZnO} / \mathrm{Si}$ diodes}

Some diodes present high values of ideality factor, probably that is caused by the occurrence of interfacial layers or surface states showing that the electrical transport is no longer governed by thermionic emission mechanism [13, 21]. In order to assess the conduction mechanism inside $\mathrm{ZnO}$ diode. The Logl-LogV characteristics are plotted within forward biasing voltage. This latter should demonstrate straight lines obeying $I \infty V^{m}$ law with diverse exponents $m$ where $m$ values vary with the injection level corresponding to the distribution of trapping centers. Furthermore, distinct linear regions are limited and fitted to extract the slopes (m) related to different conduction mechanisms like Ohmic, space-charge-limited current (SCLC), trap filling limit (TFL) or trap-free SCLC regimes.

Schematic for intrinsic point defects: vacancy, impurity interstitial, self-interstitial and substantial are described in Figure 1. The vacancy and interstitial as intrinsic point defects are displayed in Figure 2.

\section{EXPERIMENTAL TECHNIQUES}

The devices based zinc oxides are fabricated by low cost spray pyrolysis technique and front metallic contacts are thermally evaporated at low pressure vacuum. $\mathrm{ZnO}$ films have been sprayed onto $\mathrm{p}-\mathrm{Si}$ substrates from $0.1 \mathrm{~mol}$ of zinc acetate $\left(\mathrm{Zn}\left(\mathrm{CH}_{3} \mathrm{COO}\right)_{2}\right.$ $2 \mathrm{H}_{2} \mathrm{O}$ ). Firstly, the powder is dissolved in methanol and the substrate must is placed under nozzle, heated and simultaneously received the sprayed micro-droplets. During the spray process, the deposition time and substrate temperature are of $5 \mathrm{~min}$ and $350{ }^{\circ} \mathrm{C}$ respectively. The obtained Al circular contacts have a radius of $1.5 \mathrm{~mm}$ and thickness of $250 \mathrm{~nm}$. The current as a function of voltage is measured in dark and illumination conditions using Keithley source meter unit 2400. The as-grown device based on $\mathrm{ZnO}$ layer is heated by homemade heating set-up from $300 \mathrm{~K}$ to $380 \mathrm{~K}$.

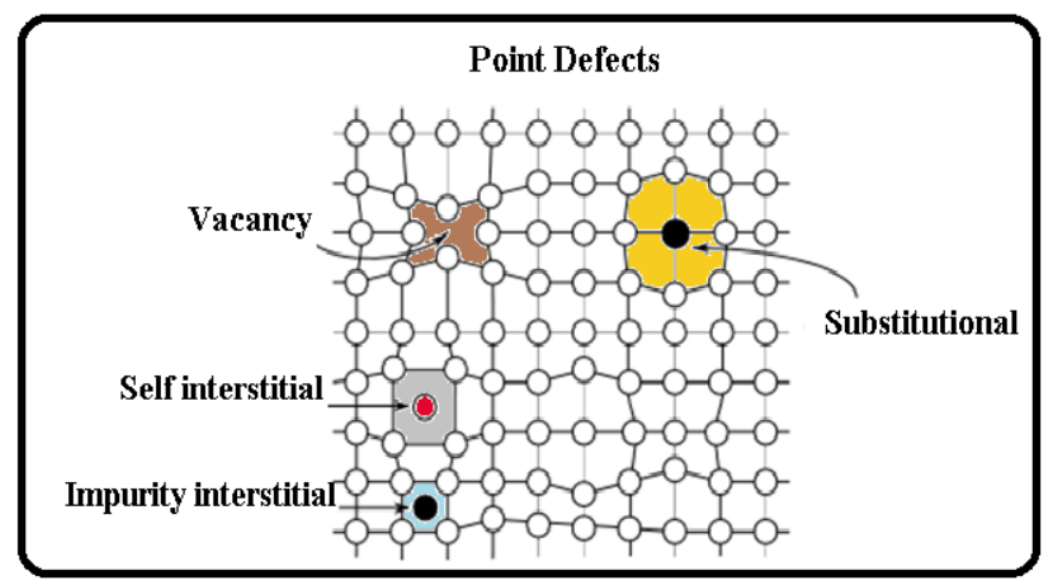

Figure 1: Schematic for intrinsic point defects: vacancy, impurity interstitial, self-interstitial and substantial.

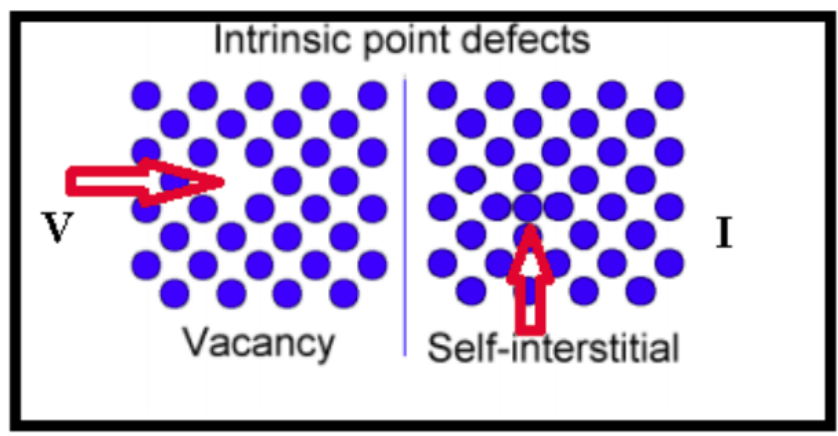

Figure 2: Schematic description of linear defects, vacancy and interstitial. 


\section{RESULTS AND DISCUSSION}

\subsection{Heterojunction Based ZnO Layer}

Nanostructured waved surface of Al doped $\mathrm{ZnO}$ films is demonstrated by 3D-scanned AFM picture in Figure 3. Schematic illustration of the $\mathrm{Al} / \mathrm{ZnO} / \mathrm{p}-\mathrm{Si} / \mathrm{Al}$ junction is drawn in Figure 4.

The current versus voltage (I-V) curves of $\mathrm{ZnO} / \mathrm{Si}$ heterojunction within $300-380 \mathrm{~K}$ temperature range is portrayed in Figure $\mathbf{5}$ showing linear (ohmic) behavior within the minor voltage range whereas a non-linear (non-ohmic) behavior at higher voltage range is occurred.
However, a non-linear behavior in I-V characteristics still dominant at greater temperatures. Linear behavior at low voltage range has been shown in the inset of Figure $\mathbf{5}$ for different temperatures. Nonohmic conduction in our material can also be verified by using relation $I \propto V^{n}$. The graph between $\ln (\mathrm{V})$ and In(I) has been plotted for all different temperatures (not shown here). The slope of the double logarithmic graph directly gives the value of exponent $n$. The value of exponent $\mathrm{n}$ is larger than one and increases with increasing temperature verifying some non-ohmic conduction mechanism in nanoparticles. This nonohmic behavior at higher field region indicates that conduction is governed by other processes like Richardson-Schottky (RS) mechanism, Poole Frenkel

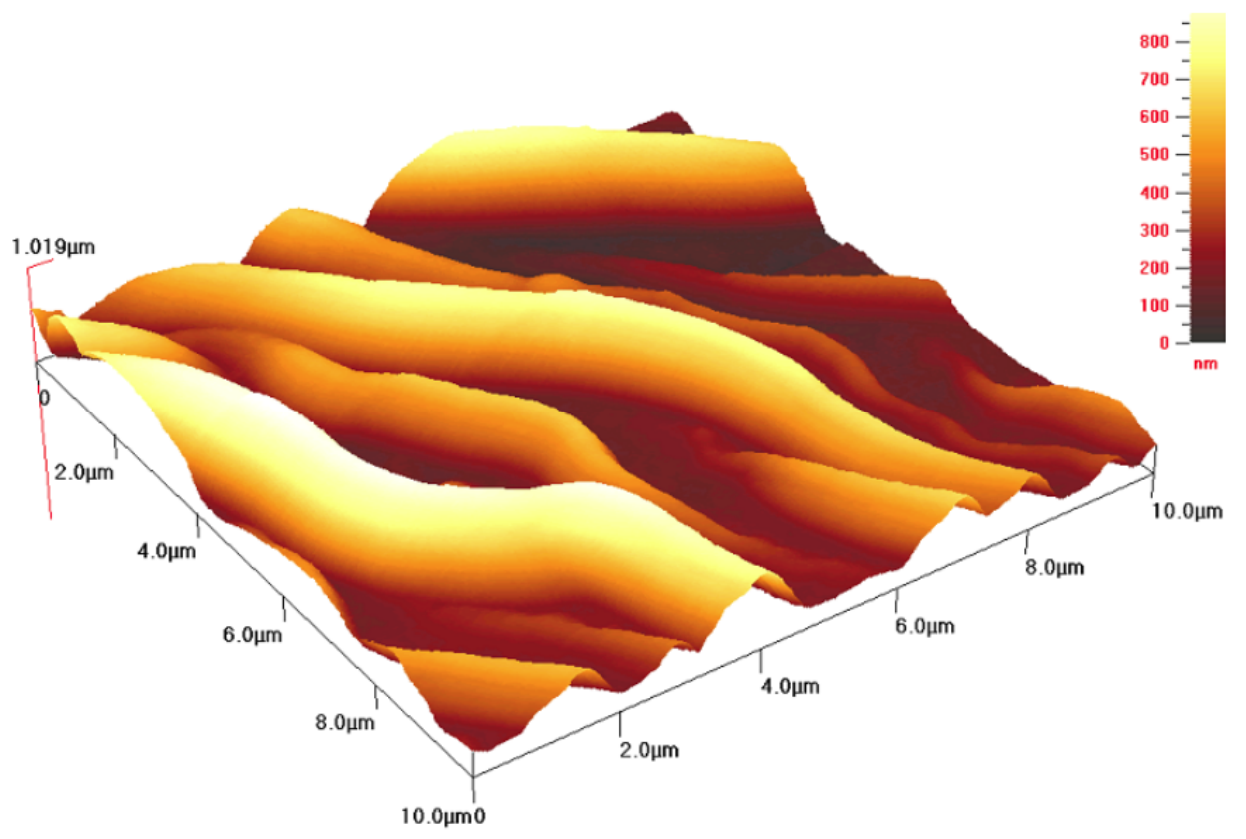

Figure 3: Al doped $\mathrm{ZnO}$ films produced by spray pyrolysis route.

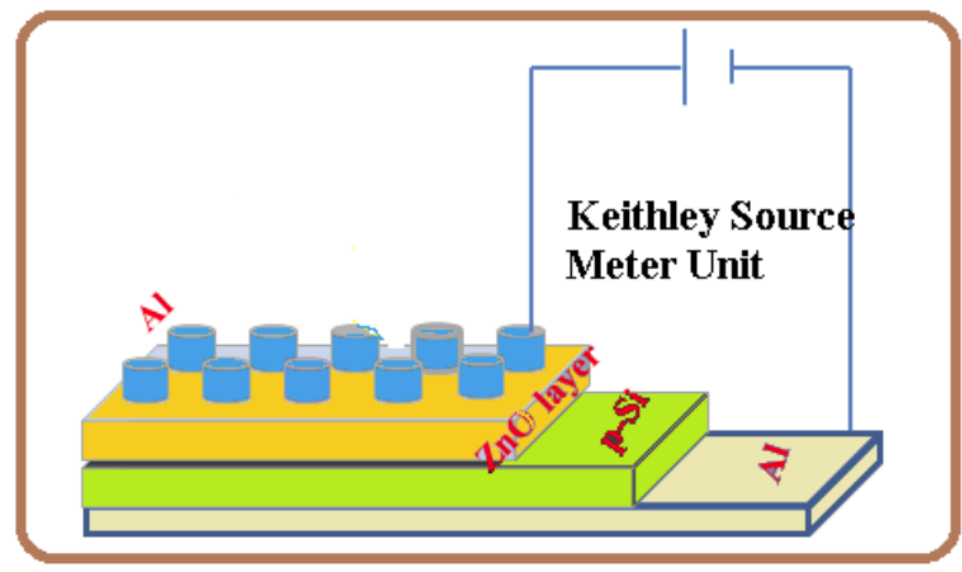

Figure 4: Schematic illustration of the $\mathrm{Al} / \mathrm{ZnO} / \mathrm{p}-\mathrm{Si} / \mathrm{Al}$ junction. 


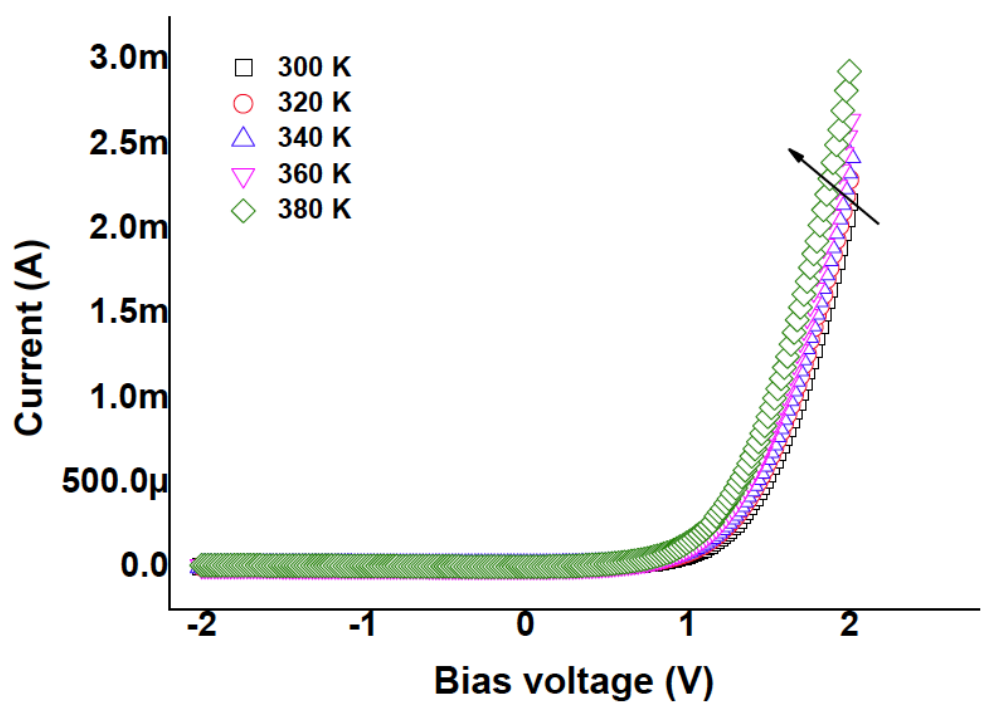

Figure 5: The current-voltage characteristics of $\mathrm{ZnO} / \mathrm{pSi}$ diode under various temperatures.

(PF) mechanism and Space charge limited current (SCLC) mechanism. To investigate high field conduction mechanism in $\mathrm{ZnO}$ nanoparticles, temperature dependent dc transport data has been further evaluated in terms of current density or conductance as a function of applied voltage or applied field. Among all the possible conduction mechanisms, the best fit to our results has been found to be PooleFrankel emission or Richardson-Schottky emission where the logarithmic conductance varies linearly with square root of the applied voltage. Slight deviation of these curves from the straight line characteristics at low voltage is observed and this has been ascribed due to contact potential effects. A thorough study on these two conduction processes has been done in past couple of decades but it is still very difficult to differentiate clearly between the Richardson-Schottky emission and the Poole-Frankel emission. Conduction owing to Richardson-Schottky emission is known as barrier or electrode limited whereas conduction because of Poole-Frenkel conductivity is bulk limited. In barrier or electrode limited, charge carriers inoculate to the material from the electrode contact by the field assisted lowering of metal semiconductor potential barrier while in bulk limited emission, charge carriers are released from traps because of field assisted lowering of depth of the trap in bulk. The Richardson-Schottky effect is related with the barrier at the surface of a metal and semiconductor. In case of RS emission process, transition of electrons from the metal electrode to the semiconducting material occurs thermionically by external applied voltage. The applied voltage lowers the barrier of diode.
Poole-Frenkel effect in fluorine doped $\mathrm{SnO}_{2}$ thin films produced by a sol-gel dip-coating technique is previously reported in which $\ln J / A T^{2}$ is plotted versus $\mathrm{V}^{1 / 2}$ at various heating temperatures. This sketch presents straight lines parts and parameters are determined like trap potential $\varphi_{\mathrm{t}}$ according to eqn.8.

A plot of $\ln J / A T^{2}$ is equivalent to $\left[\mathrm{b} / \mathrm{V}^{1 / 2}-\varphi_{t}\right] / \mathrm{k} T$ linear curve where $b=7.59 \mathrm{eV}^{1 / 2}$ is a constant [7].

As previously it is noticed that coulomb potential is distorted by external electric field. So the height of trap barrier is narrowed [22]. This happens in heterojunction which make electron migrate by thermal activation from traps to conduction band of semiconductor $[23,24]$

The PF effect is present in bulk material, and the Richardson-Schottky effect correlates to the barrier at the surface of a metal and semiconductor, according to a prior work on $\mathrm{ZnS}$.

The value of $m$ is more than unity and increases with rising temperature when using the straight parts of the $\log \mathrm{l}-\log \mathrm{V}$ curve matching the $l \alpha \mathrm{V}^{\mathrm{m}}$ law, revealing some non-ohmic conduction mechanism in nanoparticles $[25,26]$.

Other mechanisms such as Richardson-Schottky (RS), Poole Frenkel (PF), and Space charge limited current (SCLC), as cited previously by Hayat et al. and $\mathrm{Pal}$ et al, direct conduction in the higher field region [25-27]. 


\subsection{I-V Characteristics Under Dark and Heating Temperature Comportment}

The forward and reverse bias voltage of $\mathrm{ZnO} / \mathrm{Si}$ heterojunction device is studied showing the effect of temperature from high to lower temperature as indicated by arrow. The effect is slightly visible in reverse biasing whereas temperature increases current in forward biasing voltage. The logl-logV plot of $\mathrm{ZnO} / \mathrm{pSi}$ diode shows linear segments which are located and well-drawn presenting many conduction mechanisms as seen in Figure 6. The electrical behavior of device obeys roughly to I $\alpha \mathrm{V}^{\mathrm{m}}$ law where $\mathrm{m}$ is exponent related to a field and dominant conduction mechanism. Within the $300-380 \mathrm{~K}$ range the attributes for an intersection dependent on the thermionic discharge (TE) model are given as mentioned in Sze [13] where is the immersion current, is the electronic charge, is the applied voltage, term is the voltage drop across the arrangement obstruction of the intersection, is the ideality factor is the Boltzman consistent and is the total temperature. The immersion current can be gotten from the extrapolation of the forward-inclination current at a zero predisposition voltage and it is given by

Where is the viable region, $A *$ is the Richardson steady (32 Acm-2 K-2 for p-Si) [13, 28, 29] and is the zeroinclination boundary tallness which can be determined from the connection.

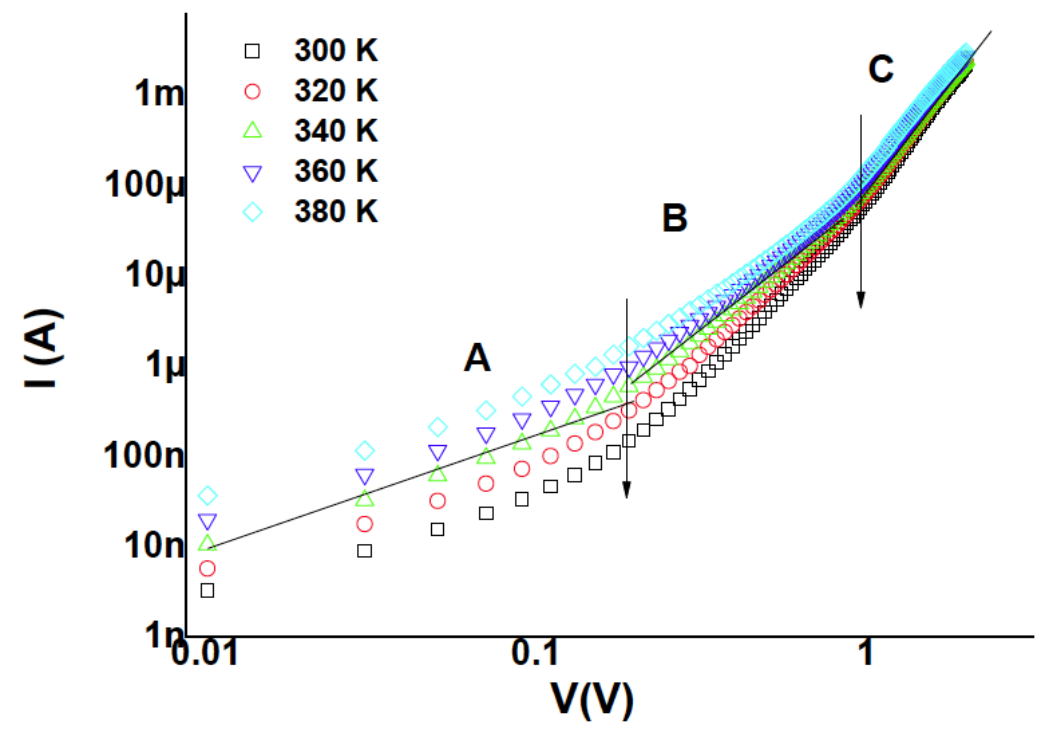

Figure 6: The log scale of current-voltage characteristics of $\mathrm{ZnO} / \mathrm{pSi}$ diode under various temperatures.

characteristics exhibit 3 regions related to three different slopes A, B and C as shown in Figure 6. Consequently three conduction regimes are happen, corresponding to linear segments for each applied heating temperature.

The first area is at low forward inclination $(0<\mathrm{V}<0.25$ $\mathrm{V})$, and the second district at higher forward predisposition $(\mathrm{V}<0.3 \mathrm{~V})$ which is ohmic. At low slope voltage, the forward current is an outstanding capacity of the applied voltage. The slant of the straight lines is discovered to be conversely relative with the expansion of temperature. Consequently, it is presumed that thermionic emission (TE) is the predominant conduction mechanism at that voltage range. The I-V

\section{CONCLUSION}

The electrical transport inside oxide-based heterojunction diodes is the subject of this research. Depending on the circumstances, such mechanisms can occur inside an insulator or semiconductor material. In reality, this study focuses on ZnO-based heterojunctions. The electrical transport in such $\mathrm{ZnO} / \mathrm{Si}$ devices is widely investigated using the average of current-voltage, capacitance, and conductance vs voltage profiles, as well as other attempts. The conduction mechanisms in the above-mentioned heterostructures are clearly described using semilog, full log functions of current. The Schottky effect is the decrease in the metal-insulator energy barrier as a 
result of electrostatic interaction with the electric field at a metal-insulator interface.

\section{ACKNOWLEDGMENTS}

The work is included in the PRFU project under contract number B00L02UN310220220001 Oran University of sciences and technology USTO-MB. www.mesrs.dz, and www.univ-usto.dz. http://www.prfumesrs.dz/. https://www.mendeley.com/impact/mostefabenhaliliba https://orcid.org/0000-0001-6507-3663.

Table 1: Nomenclature of the Used Symbols

\begin{tabular}{|c|c|}
\hline The used Symbols & Identification \\
\hline \hline $\mathrm{J}$ & Current density \\
\hline $\mathrm{E}$ & Electric field \\
\hline $\mathrm{Jn}$ & Electron current density \\
\hline$\Phi_{\mathrm{t}}$ & The trap potential \\
\hline $\mathrm{Ei}$ & Applied electric field \\
\hline $\mathrm{m}^{*}$ & Electron effective mass \\
\hline$\varphi_{\mathrm{b}}$ & Effective Schottky barrier height \\
\hline$\hbar=\mathrm{h} / 2 \pi$ & Reduced Planck constant \\
\hline $\mathrm{q}$ & Electric charge \\
\hline $\mathrm{J}$ & Current density \\
\hline
\end{tabular}

\section{REFERENCES}

[1] Handan Aydın, Fahrettin Yakuphanoglu, Cihat Aydın, Journal of Alloys and Compounds 2019; 773(30): 802-811 https://doi.org/10.1016/j.jallcom.2018.09.327

[2] M. Benhaliliba, Optik 2021; 241: 167197. https://doi.org/10.1016/j.ijleo.2021.167197

[3] XY. Zhang, RX. Xu, XY. Gao, M. Li, X.N. Shi, YD. Ji, FJ. Qian, JY. Fan, HY. Wang, WW. Li, H. Yang, Applied Surface Science 2020; 516(30): 146093. https://doi.org/10.1016/j.apsusc.2020.146093

[4] Giacomo Buccella, Davide Ceresoli, Andrea Villa, Luca Barbieri, Roberto Malgesini, Materials Chemistry and Physics 2021; 263(15): 124268. https://doi.org/10.1016/j.matchemphys.2021.124268

[5] Wenchang Zhou, Dongbin Qian, Jie Yang, Xinwen Ma, International Journal of Mass Spectrometry 2021; 462: 116516.

https://doi.org/10.1016/j.jims.2020.116516

[6] Susumu Ohki, Hiroki Funato, Michihiko Suhara, Tsugunori Okumura, Lars-Erik Wernersson, Werner Seifert, Applied Surface Science 2002; 190: 288-293. https://doi.org/10.1016/S0169-4332(01)00870-4

[7] AN. Banerjee, R. Maity, S. Kundoo, and KK. Chattopadhyay, Poole-Frenkel effect in nanocrystalline $\mathrm{SnO} 2 \mathrm{~F}$ thin films prepared by a sol-gel dip-coating technique phys. stat. sol. (a) 2004; 201(5): 983-989.

https://doi.org/10.1002/pssa.200306766
[8] Manisha Tyagi, Monika Tomar, Vinay Gupta Materials Research Bulletin 2015; 66: 123-131.

https://doi.org/10.1016/j.materresbull.2015.02.015

[9] Takuya Ogawa, Don-Chan Cho, Kazue Kaneko, Tatsuo Mori, Teruyoshi Mizutani, Thin Solid Films 2003; 438-439: 171176. https://doi.org/10.1016/S0040-6090(03)00729-6

[10] B. Tatar, AE. Bulgurcuoglu, P. Gökdemir, P. Aydogan, D. Yılmazer, O. Özdemir, K.Kutlu, Int. J. Hydrogen Energy 2009; 34: 5208. https://doi.org/10.1016/j.ijhydene.2008.10.040

[11] Jingxian Liang, Longkun Lai, Zhaokun Zhou, Jing Zhang, Jie Zhang, Jin Xu, Yipeng Zhang, Xinyu Liu, Weijun Luo, SolidState Electronics 2019; 160: 107622. https://doi.org/10.1016/j.sse.2019.107622

[12] I. Jabbari, M. Baira, H. Maaref, R. Mghaieth, Solid State Communications, 2020; 314-315: 113920. https://doi.org/10.1016/j.ssc.2020.113920

[13] KK. Ng, SM. Sze, Physics of Semiconductor Devices, third ed, John Wiley \& Sons, New Jersey, (2007).

[14] HC. Card, "Tunnelling MIS Structures," Inst. Phys. Con J Sex, 1980; 50: 140.

[15] J. Frenkel, "On the Theory of Electric Breakdown of Dielectrics and Electronic Semiconductors," Tech. Phys. USSR, 1938; 5: 685; "On Pre-Breakdown Phenomena in Insulators and Electronic Semiconductors," Phys. Rev. 1938; 54: 647. https://doi.org/10.1103/PhysRev.54.647

[16] KL. Chopra, in: Thin Film Phenomenon (McGraw-Hill, New York, 1969), p. 506.

[17] H. Zhang, EJ. Miller, ET. Yu, Analysis of leakage current mechanisms in Schottky contacts to GaN and Al0.25Ga0.75N/ GaN grown by molecular beam epitaxy. J. Appl. Phys. 2006; 99: 023703. https://doi.org/10.1063/1.2159547

[18] JR. Yeargan, HL. Taylor, The Poole-Frenkel effect with compensation present. J. Appl. Phys. 1968; 39: 12. https://doi.org/10.1063/1.1656022

[19] SE. Meftah, M. Benhaliliba, M. Kaleli, CE. Benouis, CA. Yavru, AB. Bayram, Physica B: Condensed Matter 2020 593(15): 412238 https://doi.org/10.1016/j.physb.2020.412238

[20] VL. Rideout, "A Review of the Theory and Technology for Ohmic Contacts to Group 111-V Compound Semiconductors," Solid-State Electron, 1975; 18: 541. https://doi.org/10.1016/0038-1101(75)90031-3

[21] JP. Colinge, CA. Colinge ISBN-13: 9780387285238Springer; 2002nd edition (October 3, 2005).

[22] AM. Katzenmeyer, F. Léonard, AA. Talin, PS. Wong, and DL. Huffaker, Nano Lett. 2010; 10: 4935. https://doi.org/10.1021/nl102958g

[23] JG. Simmons, Phys. Rev. 155, 657 (1967) J. G. Simmons, Phys. Rev. 1967; 155: 657. https://doi.org/10.1103/PhysRev.155.657

[24] M. El-Samanoudy, Applied Surface Science 207, 219 (2003) M. El-Samanoudy, Applied Surface Science 2003; 207, 219. https://doi.org/10.1016/S0169-4332(02)01365-X

[25] Z. Imran, M. Rafiq, and M. Hasan, AIP Advances 44, 067137 (2014). $22 \mathrm{~K}$. https://doi.org/10.1063/1.4885462

[26] Hayat, M. Rafiq, A. ur Rahman, AA. Khan, and M. Hasan, Progress in Natural Science: Materials International 2013; 23: 388. https://doi.org/10.1016/j.pnsc.2013.06.016

[27] A. Pal and P. Khare, Journal of Electrostatics 2013; 71: 976. https://doi.org/10.1016/j.elstat.2013.09.005 
[28] MM. El- Nahass, MM. Abd El-Raheem, AA. Atta, AM. Hassanien, Radiat. Phys. Chem. 2014; 103: 227. https://doi.org/10.1016/j.radphyschem.2014.05.055
[29] MM. El-Nahassa, AAM. Farag, Mossad El-Metwally, F.S.H. Abu Samaha, Eman Elesh, Synth. Met 2014; 195: 110. https://doi.org/10.1016/j.synthmet.2014.05.013

Received on 29-11-2021

DOI: https://doi.org/10.31875/2409-9694.2021.08.6

(c) 2021 M. Benhaliliba; Licensee Zeal Press.

This is an open access article licensed under the terms of the Creative Commons Attribution Non-Commercial License (http://creativecommons.org/licenses/by-nc/3.0/), which permits unrestricted, non-commercial use, distribution and reproduction in any medium, provided the work is properly cited. 